\title{
Electromagnetic navigation bronchoscopy fiducial marker margin identification plus triple dye for complete lung nodule resection
}

\author{
Joyce W. Y. Chan, MBBS(Hons), MRCS, Rainbow W. H. Lau, MBChB, FRCS, and
} Calvin S. H. Ng, MD, FRCS, Hong Kong SAR, China

\footnotetext{
From the Division of Cardiothoracic Surgery, Department of Surgery, Prince of Wales Hospital, The Chinese University of Hong Kong, Hong Kong SAR, China.

Disclosures: Dr Ng is a consultant for Johnson \& Johnson and Medtronic, USA. All other authors reported no conflicts of interest.

The Journal policy requires editors and reviewers to disclose conflicts of interest and to decline handling or reviewing manuscripts for which they may have a conflict of interest. The editors and reviewers of this article have no conflicts of interest.

Received for publication July 6, 2020; revisions received July 6, 2020; accepted for publication July 8, 2020; available ahead of print July 11,2020

Address for reprints: Calvin S. H. Ng, MD, FRCS, Division of Cardiothoracic Surgery, Department of Surgery, The Chinese University of Hong Kong, Prince of Wales Hospital, Hong Kong SAR, China (E-mail: calvinng@surgery.cuhk.edu.hk).

JTCVS Techniques 2020;3:329-33

2666-2507

Copyright (C) 2020 The Authors. Published by Elsevier Inc. on behalf of The American Association for Thoracic Surgery. This is an open access article under the CC BY-NC-ND license (http://creativecommons.org/licenses/bync-nd/4.0/)

https://doi.org/10.1016/j.xjtc.2020.07.010
}

Video clip is available online.

Successful sublobar resection of lung nodules during videoassisted thoracic surgery (VATS) requires not only accurate localization of target lesions but also adequate resection margins. Electromagnetic navigation bronchoscopy (ENB) dye marking of lesions has become a useful tool for lung nodule localization. However, due to difficulty of palpating small lung nodules or ground-glass opacities during VATS, and lack of lesion depth perception even with dye marking, intraoperative determination of resection margin is still problematic. With patients' consent to present their case histories and relevant imaging, we share our technique of fiducial marker placement via ENB, allowing confirmation of adequate resection margin during immediate subsequent surgery.

\section{TECHNIQUE DESCRIPTION}

In the hybrid operation room (HOR), ENB with fluoroscopy and cone-beam computed tomography (CBCT) guidance is used to reach the lung lesion. A fine-needle aspiration needle is inserted and CBCT shows needle tip at

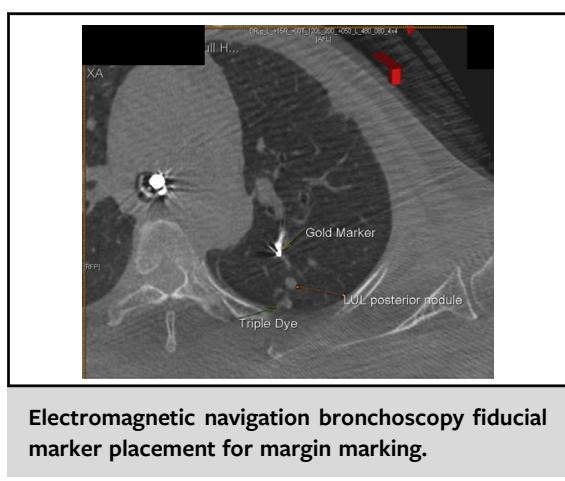

\begin{abstract}
CENTRAL MESSAGE
Fiducial marker placement and triple contrast dye marking of lung lesions during electromagnetic navigation bronchoscopy provides accurate localization and ensures adequate resection margin.
\end{abstract}

See Commentaries on pages 334 and 336.

target lesion (Figure 1). To aid subsequent VATS identification of the lesion, the needle is advanced to a subpleural location under fluoroscopy, and $0.3 \mathrm{~mL}$ of triple contrast is injected (equal volumes of iohexol imaging contrast, methylene blue, and indocyanine green) (Video 1). ${ }^{1}$ Subsequently, the extended working channel is retracted to the desired deep margin distance from the lesion as measured on CBCT images, where a SuperLock (Medtronic, Minneapolis, Minn) gold-nitinol coil fiducial marker is placed, with its position confirmed by CBCT (Figure 2 and Video 2).

In same HOR session, the single lumen endotracheal tube is exchanged for double-lumen tube for VATS. Upon entering pleural space, the target lesion can be identified using methylene blue and indocyanine green under white light and near-infrared light, respectively (Figure 3,A). The 


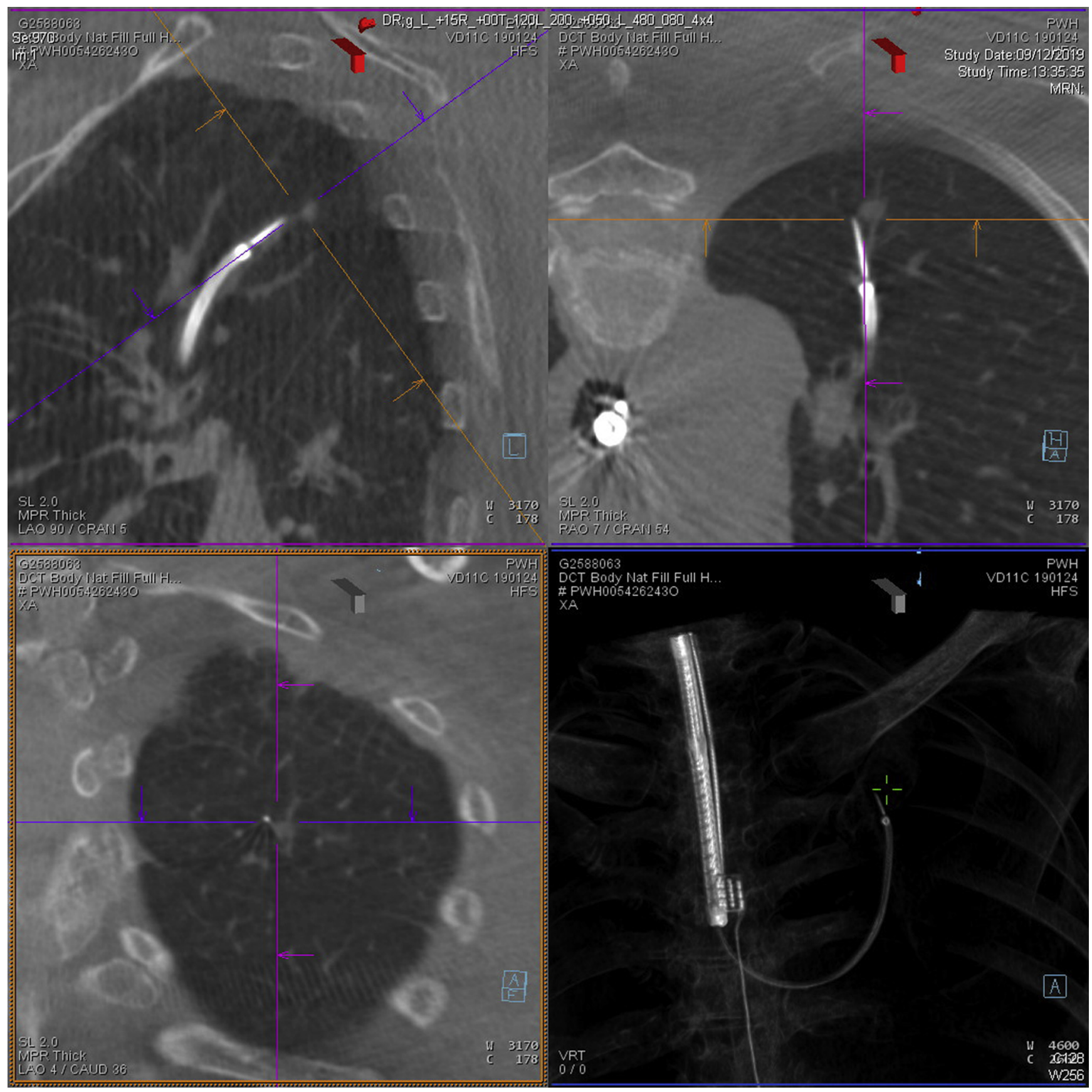

FIGURE 1. Cone beam computed tomography on 3 planes in the hybrid operating room demonstrates accurate positioning of electromagnetic navigation bronchoscopy-guided needle tip in close proximity to the left upper lobe lung nodule.

intended staple line is gently clamped with a Foerster lung clamp, and fluoroscopy confirms inclusion of the fiducial marker, followed by stapled wedge resection of the lesion (Figure 3, B, and Videos 3 and 4). Presence of marker at the specimen confirms lesion excision with sufficient margin (Figure 3, C).

\section{DISCUSSION}

For non-anatomical sublobar resection, margins are important because tumor satellite cells are found in surrounding macroscopically normal lung tissues, and presence of spread through air-spaces occur most commonly within $2 \mathrm{~mm}$ of tumors. ${ }^{2}$ While studies demonstrated an 


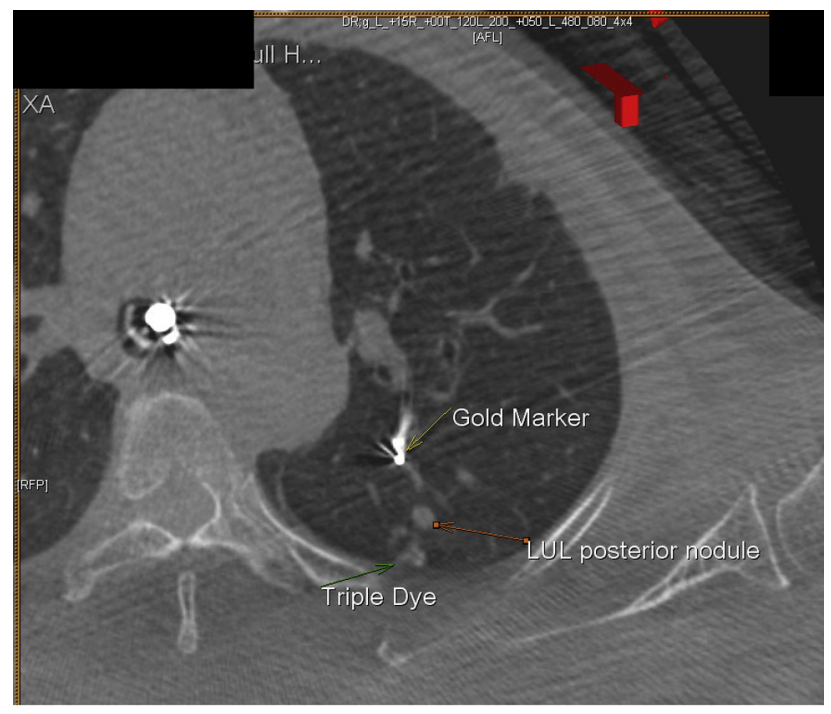

FIGURE 2. Cone beam computed tomography in the hybrid operating room showing posterior left upper lobe target lung nodule, triple dye containing iohexol contrast, methylene blue, and indocyanine green at subpleural location, and gold fiducial marker at $1 \mathrm{~cm}$ from lung nodule at the desired resection margin. $L U L$, Left upper lobe. inverse relationship between margin distance and recurrence rate, there is no consensus regarding the exact margin required. Proposed criteria include margin distance at least maximal tumor size for non-small cell carcinoma, ${ }^{3}$ or $8-\mathrm{mm}$ margin for lung metastasis $>1 \mathrm{~cm}$ size. $^{2}$

Our institute has been using ENB dye marking in HOR for intraoperative lung lesion localization with satisfactory results. ${ }^{4}$ However, if the lesion is located at a distance away from the pleura, injecting dye into the lesion itself may result in difficulty in identifying the dye on pleural surface during VATS. Injecting a larger volume of dye, on the contrary, may result in large area of "coloring" on pleural surface, making precise localization problematic. Therefore, our technique injects a small volume of dye, usually 0.3 to $0.5 \mathrm{~mL}$, to a subpleural location past the actual position of the lesion. During VATS, the location of the lesion can be conceptualized to be vertically beneath pleural surface at a certain distance.

Deeper lesions that are small or of ground glass texture also pose difficulty in ensuring adequate

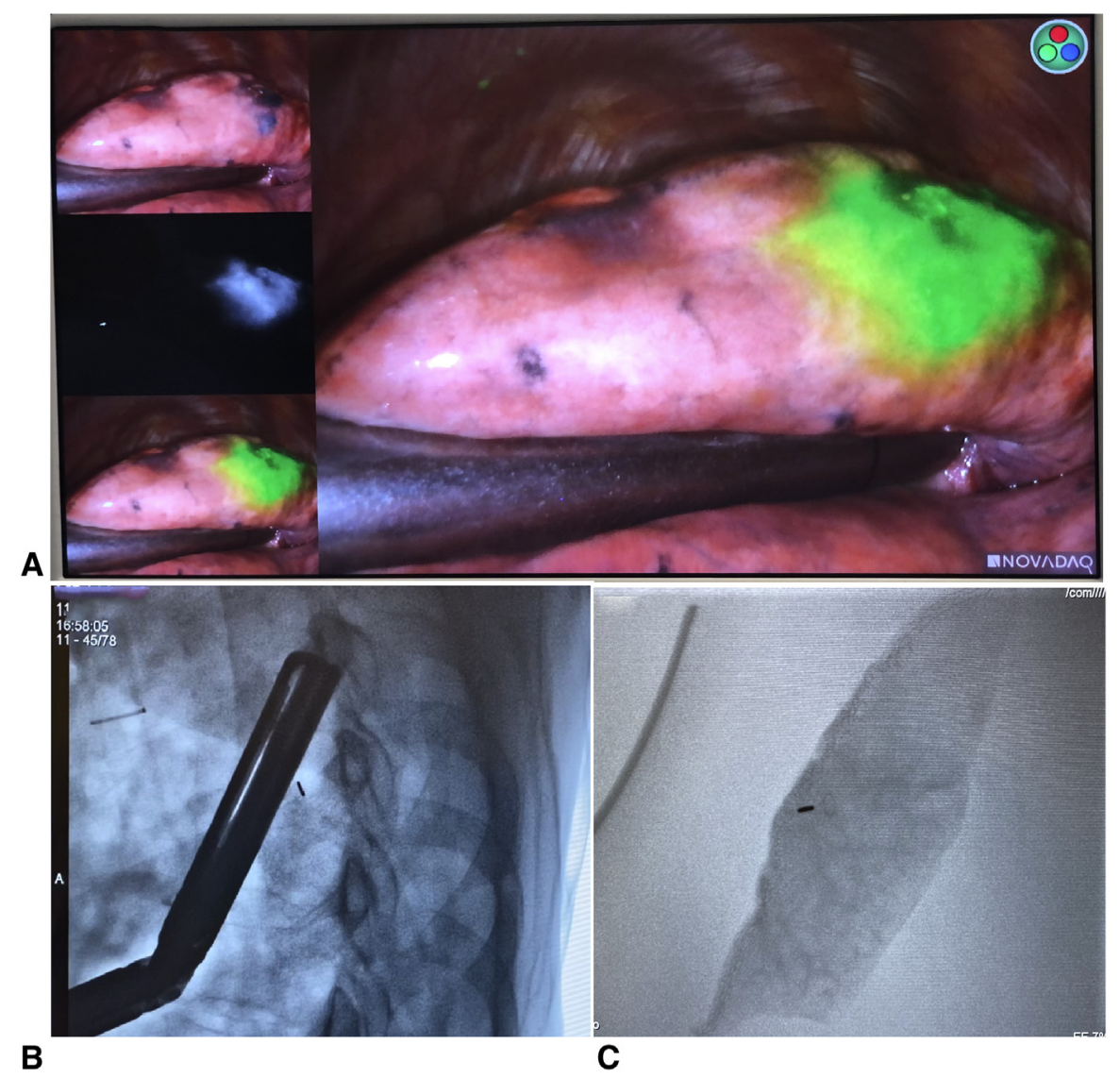

FIGURE 3. A, Target lesion localization during video-assisted thoracic surgery after triple contrast dye marking. Top of left panel shows methylene blue marking on pleural surface under normal lighting, whereas the other panels show fluorescent indocyanine green markings under various modes of near infrared light. B, Stapling across resection margin under live fluoroscopy with a metal fiducial marker on the specimen side. C, Fluoroscopy image taken in the hybrid operating room of resected wedge specimen with fiducial marker inside and staple line immediately to marker's left. 


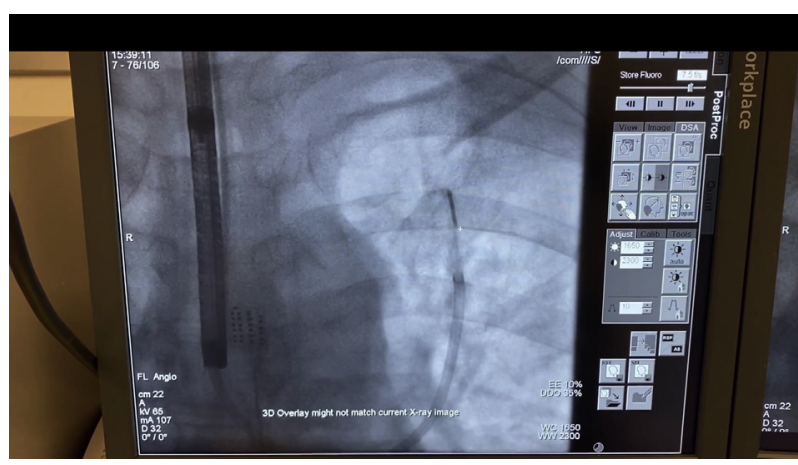

VIDEO 1. Our triple contrast dye containing iohexol contrast is injected into the lung nodule through a fine needle via the extended working channel of the electromagnetic navigation bronchoscope. Video available at: https://www.jtcvs.org/article/S2666-2507(20)30348-5/fulltext.

resection margin. In the past, we empirically perform a large wedge resection of the dyed lung parenchyma with a mental estimation of the margin required. This method is imprecise, and margin can only be ascertained after the specimen is resected and cut open. A larger than required margin sacrifices lung function and threatens large vessels or bronchi near the hilar region, whereas a smaller than desired margin may necessitate further resection, which is troublesome, time-consuming and staple-consuming.

The SuperLock fiducial marker was designed for ENB placement to guide radiation therapy. ${ }^{5}$ We expanded its usage to guide subsequent surgical resection, as it solves the margin issue by completing 3-dimensional localization of lung lesion. A wedge resection of the lung is often dome-shaped on computed tomography, with the crown of dome marked by dye and center base of

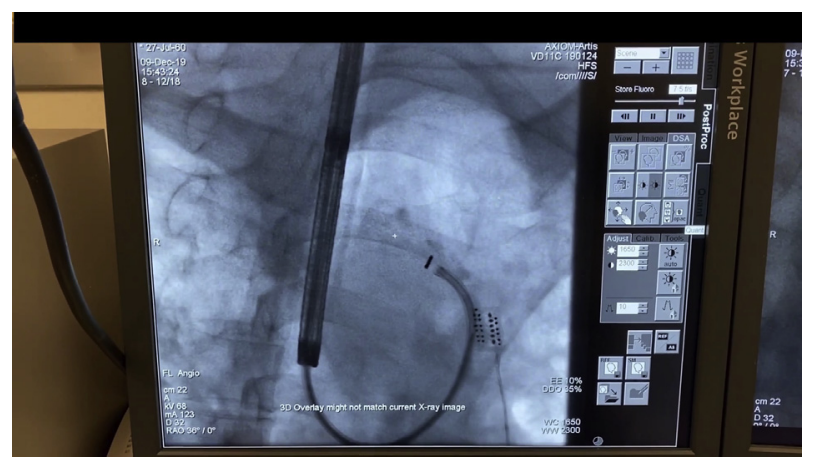

VIDEO 2. A SuperLock (Medtronic, Minneapolis, MN) gold-nitinol coil fiducial marker is deployed at the desired margin via the extended working channel of the electromagnetic navigation bronchoscope. Video available at: https://www.jtcvs.org/article/S2666-2507(20)30348-5/fulltext.

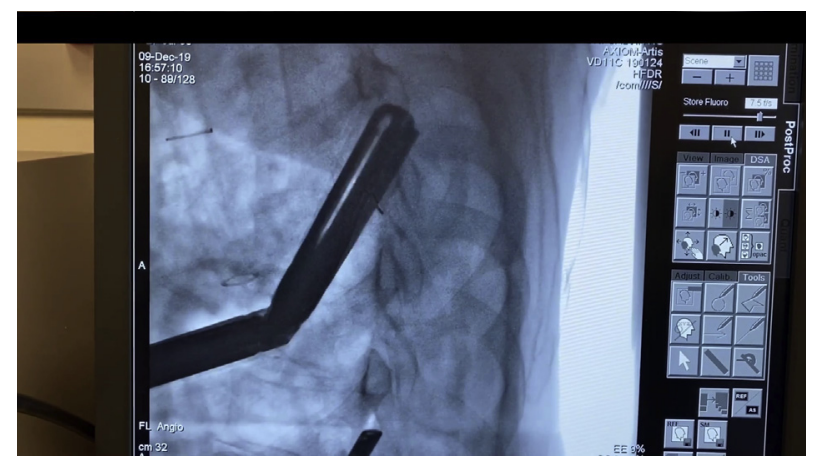

VIDEO 3. In the hybrid operating room, fluoroscopy is used during surgery to confirm inclusion of the fiducial marker on the specimen side (in this case, the marker is to the right of the stapler, which is gently clamped at the desired margin). Video available at: https://www.jtcvs. org/article/S2666-2507(20)30348-5/fulltext.

dome marked by fiducial marker. Adjustment of stapler across the margin is easy with fluoroscopy to confirm inclusion of fiducial marker on specimen side. The placement of fiducial marker during ENB allows precise design of margin on computed tomography without any parenchymal distortion. ${ }^{2,3}$ Although this technique is generally safe and reliable, complications like bleeding, pneumothorax, or marker migration may occur, albeit low in literature. ${ }^{5}$

The combination of our dye-marking technique and placement of fiducial marker can be performed easily and quickly by ENB, typically requiring only 5 minutes each after successful navigation. This approach is most useful for selected cases of deep and small malignant lung lesions where adequate margin is essential for reducing local recurrence.

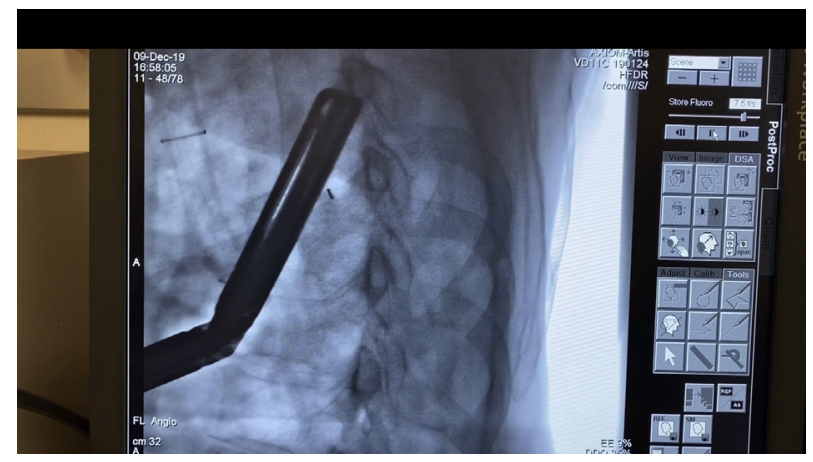

VIDEO 4. Fluoroscopy showing stapling at the desired margin such that the marker is on the specimen side. Video available at: https://www. jtcvs.org/article/S2666-2507(20)30348-5/fulltext. 


\section{References}

1. Ng C, Zhao Z, Long H, Lau R. Electromagnetic navigation bronchoscopy triple contrast dye marking for lung nodule localization. Thorac Cardiovasc Surg. 2019;68:253-5.

2. Welter S, Barile La Raia R, Gupta V. Pursuit of an optimal surgical margin in pulmonary metastasectomy. J Visualized Surg. 2019;5:39.

3. Sawabata N, Ohta M, Matsumura A, Nakagawa K, Hirano H, Maeda H, et al. Optimal distance of malignant negative margin in excision of nonsmall cell lung cancer: a multicenter prospective study. Ann Thorac Surg. 2004;77:415-20.

4. Ng CSH, Chu CM, Lo CK, Lau RWH. Hybrid operating room Dynacomputed tomography combined image-guided electromagnetic navigation bronchoscopy dye marking and hookwire localization video-assisted thoracic surgery metastasectomy. Interact Cardiovasc Thorac Surg. 2018; 26:338-40.

5. Bowling MR, Folch EE, Khandhar SJ, Kazakov J, Krimsky WS, LeMense GP, et al. Fiducial marker placement with electromagnetic navigation bronchoscopy: a subgroup analysis of the prospective, multicenter NAVIGATE study. Ther Adv Respir Dis. 2019;13:1753466619841234. 